\title{
Self-imaging with finite energy
}

\author{
Rafael Piestun, Yoav Y. Schechner, and Joseph Shamir
}

Department of Electrical Engineering, Technion-Israel Institute of Technology, Haifa 32000, Israel

Received September 20, 1996

\begin{abstract}
General solutions and conditions are presented for paraxial waves that image themselves with different scales through free propagation. These waves, represented as superpositions of Gauss-Laguerre modes, have finite energy and thus finite effective width. The self-imaging wave fields described by Montgomery [J. Opt. Soc. Am. 57, 772 (1967)], which possess a Fourier transform that is confined to a ring structure, are obtained as a specific limiting case of an infinite aperture. (C) 1997 Optical Society of America
\end{abstract}

Wave fields possessing invariance and conservation properties have recently stimulated research activity. Typical examples of such fields are Gaussian modes, Bessel beams, ${ }^{1}$ phase dislocations, ${ }^{2}$ and beams with rotating intensity distributions. ${ }^{3}$ The subject of this Letter is self-imaging (SI) in which the invariance property is manifested in a repetition of the transverse intensity distribution of a wave along the direction of propagation. Montgomery $y^{4}$ was the first to derive general conditions for a coherent wave field to have its complex amplitude repeated along the illumination direction. His results are valid for the homogeneous Helmholtz equation and require an infinite support of the wave on any transverse plane. Moreover, every solution satisfying these conditions carries infinite energy outside any finite support. In Ref. 5 it was shown that for finite-energy waves this SI has an approximate character. Other studies ${ }^{6-9}$ considered the finiteness of object dimensions as an aberrating factor. Furthermore, they assumed transversal periodicity of the object, which is an important but by no means a general case. In Refs. 1 and 10 a superposition of nondiffracting beams was used for describing SI waves.

Here we seek general paraxial waves that carry finite total power and exhibit scaled SI; i.e., the repeated images can be scaled relative to each other. The formalism is based on an expansion in GaussLaguerre (GL) or Gauss-Hermite (GH) propagation modes. In this way we obtain simple yet powerful conditions on the general structure of paraxial waves possessing scaled SI. It is also shown that the scaled phase map is self-imaged except for a quadratic-radial phase term. The known case of an infinite aperture is obtained as a limiting case.

Let a scalar wave be represented by the function

$$
f(\mathbf{r}, t)=u(\mathbf{r}) \exp [i(k z-\omega t)],
$$

where $\mathbf{r}=(\rho, \phi, z)$ in cyclindrical coordinates, $\omega$ is the angular frequency, and $k$ is the wave number. The reduced wave field, $u(\mathbf{r})$, can be expanded in terms of GL modes. We found it convenient to write each GL mode in the form

$$
u_{n, m}(\mathbf{r})=G(\tilde{\rho}, \tilde{z}) R_{n, m}(\tilde{\rho}) \Phi_{m}(\phi) Z_{n}(\tilde{z}),
$$

where $\tilde{\rho}=\rho / w(\tilde{z})$ is the radial coordinate scaled by the Gaussian spot size, which is given by $w(\tilde{z})=$ $w_{0}\left(1+\tilde{z}^{2}\right)^{1 / 2}$. We defined $\tilde{z} \equiv z / z_{0}$ as the longitudinal coordinate scaled by the Rayleigh length, $z_{0}=\pi w_{0}^{2} / \lambda$. The functions comprising $u_{n, m}(\mathbf{r})$ are

$$
\begin{aligned}
G(\tilde{\rho}, \tilde{z}) & =\frac{w_{0}}{w(\tilde{z})} \exp \left(-\tilde{\rho}^{2}\right) \exp \left(i \tilde{\rho}^{2} \tilde{z}\right) \exp [-i \psi(\tilde{z})], \\
R_{n, m}(\tilde{\rho}) & =(\sqrt{2} \tilde{\rho})^{|m|} L_{(n-|m|) / 2}^{|m|}\left(2 \tilde{\rho}^{2}\right), \\
\Phi_{m}(\phi) & =\exp (i m \phi), \\
Z_{n}(\tilde{z}) & =\exp [-i n \psi(\tilde{z})],
\end{aligned}
$$

while $\psi(\tilde{z})=\arctan (\tilde{z})$ is the Gouy phase. The function (2b) is common to all modes and comprises the radial Gaussian envelope of the beam, a quadratic phase, and a Gouy phase. $L_{(n-|m|) / 2}^{|m|}$ are the generalized Laguerre polynomials, where the integers $n, m$ satisfy

$$
n=|m|, \quad|m|+2, \quad|m|+4, \ldots .
$$

Let us examine a superposition of such modes:

$$
u(\mathbf{r})=\sum_{j=1}^{S} A_{j} u_{n_{j}, m_{j}}(\mathbf{r}),
$$

where $A_{j}$ is the complex amplitude of mode $j$. Assuming, without loss of generality, that $n_{j} \leq n_{j+1}$, the intensity distribution is given by

$$
\begin{aligned}
I(\mathbf{r})= & |G(\tilde{\rho})|^{2}\left\{\sum_{j=1}^{S}\left|A_{j}\right|^{2} R_{n_{j}, m_{j}}^{2}(\tilde{\rho})\right. \\
& +\sum_{j=1}^{S} \sum_{p=j+1}^{S} 2\left|A_{j}\right|\left|A_{p}\right| R_{n_{j}, m_{j}}(\tilde{\rho}) \\
& \left.\times R_{n_{p}, m_{p}}(\tilde{\rho}) \cos \left[\Delta m_{j p} \phi-\Delta n_{j p} \psi(\tilde{z})-\vartheta_{j p}\right]\right\},
\end{aligned}
$$

where $\Delta m_{j p} \equiv m_{j}-m_{p}, \Delta n_{j p} \equiv n_{j}-n_{p}$, and $\vartheta_{j p} \equiv$ $\left[\arg \left(A_{j}\right)-\arg \left(A_{p}\right)\right]$. Considering SI scaled as the elementary Gaussian spot size, we require that

$$
I\left(\tilde{\rho}, \phi, z_{1}\right)=I\left(\tilde{\rho}, \phi+2 \pi N, z_{2}\right) \text { for all } \tilde{\rho}, \phi .
$$

The first sum on the right-hand side of Eq. (5) is invariant on propagation along $\tilde{z}$ (except for scale) and is therefore self-imaged. Each term in the second sum represents a wave rotating linearly with 
$\psi(\tilde{z}){ }^{3} \quad$ Accordingly, Eq. (6) will be fulfilled if each of these terms is equal at $z_{1}$ and $z_{2}$ :

$$
\begin{aligned}
& \cos \left[\Delta m_{j p} \phi-\Delta n_{j p} \psi\left(\tilde{z}_{1}\right)-\vartheta_{j p}\right] \\
& \quad=\cos \left[\Delta m_{j p}(\phi+2 \pi N)-\Delta n_{j p} \psi\left(\tilde{z}_{2}\right)-\vartheta_{j p}\right] .
\end{aligned}
$$

The coefficients of the cosine functions depend on $\tilde{\rho}$ through the functions $R_{n_{j}, m_{j}}(\tilde{\rho}) R_{n_{p}, m_{p}}(\tilde{\rho})$. Thus the equality of each term, and hence Eq. (7), is also a necessary (as well as sufficient) condition for the fulfillment of Eq. (6). Equation (7) is satisfied for all $\phi$ if and only if

$$
\begin{aligned}
& \Delta m_{j p} \phi-\Delta n_{j p} \psi\left(\tilde{z}_{1}\right)-\vartheta_{j p} \\
& \quad=\left[\Delta m_{j p}(\phi+2 \pi N)-\Delta n_{j p} \psi\left(\tilde{z}_{2}\right)-\vartheta_{j p}\right]+2 \pi N^{\prime}
\end{aligned}
$$

for all $\phi$. We are led to the condition that

$$
\Delta n_{j p}=N_{j p} \Omega\left(\tilde{z}_{2}, \tilde{z}_{1}\right) \quad \text { for all modes } j, p,
$$

where $N_{j p}$ is any integer and

$$
\Omega\left(\tilde{z}_{2}, \tilde{z}_{1}\right) \equiv \frac{2 \pi}{\psi\left(\tilde{z}_{2}\right)-\psi\left(\tilde{z}_{1}\right)}=\frac{2 \pi}{\Delta \psi} .
$$

Equation (9) is fulfilled if and only if the index $n_{j}$ of each mode $j$ that comprises the wave satisfies

$$
n_{j}=n_{1}+N_{j} \Omega\left(\tilde{z}_{2}, \tilde{z}_{1}\right) \text { for all } j,
$$

where $n_{1}$ and $N_{j}$ are integers. The constant $n_{1}$ is arbitrary, whereas the variable $N_{j}$ is such that $n_{j}$ is a nonnegative integer. The magnification between selfimages is

$$
M=w\left(\tilde{z}_{2}\right) / w\left(\tilde{z}_{1}\right)=\left\{\left[1+\left(\tilde{z}_{2}\right)^{2}\right] /\left[1+\left(\tilde{z}_{1}\right)^{2}\right]\right\}^{1 / 2} .
$$

Although we required SI between two planes, Eq. (5) indicates that the scaled intensity distribution is periodic in $\psi(\tilde{z})$. Because $\psi(\tilde{z})$ is bounded, the number of periods is finite. Note that a SI condition between two planes was also sought in Ref. 4, leading to periodic solutions in $z$.

We now investigate the phase relation between these intensity self-images. The amplitude of the wave presented in Eq. (4) is

$$
\begin{aligned}
u(\mathbf{r})= & \frac{w_{0}}{w(\tilde{z})} \exp \left[-i\left(1+n_{1}\right) \psi(\tilde{z})\right] \\
& \times \exp \left(i \tilde{\rho}^{2} \tilde{z}\right) \sum_{j=1}^{M} A_{j} K_{n_{j}, m_{j}}(\tilde{\rho}) \Phi_{m_{j}}(\phi) \\
& \times \exp \left[-i\left(n_{j}-n_{1}\right) \psi(\tilde{z})\right]
\end{aligned}
$$

where $K_{n_{j}, m_{j}}(\tilde{\rho})$ comprises all the scaled-radial factors that do not depend explicitly on $\tilde{z}$. Because of the orthogonality of the modes, the sum is equal in $z_{1}$ and $z_{2}$ if and only if each term in it is equal in these two planes. This condition is equivalent to Eq. (8). Thus the two-dimensional scaled phase distributions are related by

$$
\begin{aligned}
\operatorname{phase}\left(\tilde{z}_{2}\right)= & \operatorname{phase}\left(\tilde{z}_{1}\right)-\left(1+n_{1}\right)\left[\psi\left(\tilde{z}_{2}\right)-\psi\left(\tilde{z}_{1}\right)\right] \\
& +\left(\tilde{z}_{2}-\tilde{z}_{1}\right) \tilde{\rho}^{2} .
\end{aligned}
$$

Hence the phase map of the wave (scaled with the Gaussian spot size) is self-imaged with the intensity distribution, except for a quadratic-radial term and a constant. Moreover, we can see that this derivation leads directly to the previous result [Eqs. (10) and (11)].
The conditions of Eqs. (10) and (11) apply for every paraxial wave showing SI with any magnification $(M)$ and between any two planes (separated by $\Delta z \equiv z_{2}-$ $z_{1}$ ). To prove this, we must find at least one suitable GL basis in which these conditions can be applied, i.e., a location for the waist and its radius. If $M=1$, then, from Eq. (12), $z_{1}=-\Delta z / 2$ and $z_{0}$ can be arbitrarily chosen. Otherwise, we obtain

$$
z_{0}^{2}=\left[\Delta z^{2}+2 z_{1} \Delta z-\left(M^{2}-1\right) z_{1}^{2}\right] /\left(M^{2}-1\right),
$$

which defines a parabolic segment on the $\left(z_{1}, z_{0}\right)$ plane. Because $\Omega\left[\left(z_{1}+\Delta z\right) / z_{0}, z_{1} / z_{0}\right]$ is continuous and nonconstant in this domain, it is always possible to choose $z_{0}$ to make $\Omega$ a rational number, which is a necessary condition for the fulfillment of Eq. (11). Note, however, that there may still be a great deal of freedom in analyzing and synthesizing the SI objects, because there are an infinite number of legitimate values of $z_{0}$ and $z_{1}$.

The solutions given by Eqs. (10) and (11) are not described by the Montgomery rings that were used to represent the general solution for SI in the literature. ${ }^{4,5}$ However, we now show that these solutions can be obtained as a special limiting case in which the beam appears to have an infinite effective extent. First we note that the paraxial SI objects introduced by Montgomery are described in their most general way by rings in the Fourier domain as

$$
\xi^{2}=\xi_{1}^{2}+(2 / \lambda \Delta z) N,
$$

where $N$ is an integer and $\xi_{1}^{2}$ is a constant describing the lowest-frequency ring. The condition presented in Ref. 4 is obtained for $\xi_{1}^{2}=0$. We set the following conditions for the mode superposition of Eq. (4):

(A) To make the modes resemble (infinite) plane waves and Bessel modes, we can reduce the quadraticradial phase by requiring that $\tilde{z} \ll 1$. In the limit $\left(\Delta z / z_{0}\right) \rightarrow 0$, we obtain a uniform periodicity in $z$ and an infinite number of self-images. According to this limit and Eq. (11),

$$
n_{j}-n_{1} \cong 2 \pi N_{j}\left(z_{0} / \Delta z\right) \stackrel{\tilde{z} \rightarrow 0}{\longrightarrow} \infty .
$$

(B) The effective half-angular beam spread of a GL mode obeys ${ }^{3}$

$$
(\pi / \lambda) \tan \left(\theta_{\text {beam }}\right)=\sqrt{n+1} / w_{0} \stackrel{n \gg 1}{\longrightarrow} \sqrt{n} / w_{0} .
$$

The angular beam spread must be kept small to satisfy the paraxial approximation, in spite of Eq. (17). For convenience we keep

$$
\lambda \sqrt{n_{j}} / w_{0}=\operatorname{constant}(j)>0 \text { for all } j
$$

during the limiting process. As a consequence, $\left(w_{0} / \lambda\right) \rightarrow \infty$, and thus also $n_{1} \rightarrow \infty$.

(C) Obviously, we demand that $\rho$ be much smaller than the effective width of the beam, which is taken as the standard deviation of the intensity distribution. It can be shown ${ }^{3}$ that

$$
\left\langle(\Delta x)^{2}\right\rangle=(1 / 4)(n+1) w^{2}(\tilde{z}) \stackrel{\tilde{z} \ll 1, n \gg 1}{\longrightarrow} n w_{0}^{2} / 4 .
$$

Thus $\tilde{\rho} \ll \sqrt{n} / 2$ for all $n$ comprising the wave. 
(D) The smaller the difference $n-|m|$, the lower the energy concentrated about the axis [where condition (C) is valid]. Thus, to maintain a significant amount of energy in the axial region, we require that $n-|m| \approx n$ (see Eq. (3)].

$$
\begin{aligned}
& \text { Using the relation }{ }^{11} \\
& \qquad \eta^{-a} L_{\eta}{ }^{a}(\chi / \eta) \stackrel{\eta \rightarrow \infty}{\longrightarrow} \chi^{-a / 2} J_{a}(2 \sqrt{\chi}),
\end{aligned}
$$

where $J_{a}$ denotes a Bessel function of order $a$, we note that conditions (A) $-(\mathrm{D})$ with Eqs. (2) and (11) lead to

$$
\begin{aligned}
u_{n, m}^{\operatorname{limit}}(\mathbf{r})= & c(n, m) \exp \left(-\frac{\rho^{2}}{w_{0}^{2}}\right) \\
& \times J_{|m|}\left\{2 \pi \rho\left[\xi_{1}^{2}+\left(\frac{2}{\lambda \Delta z}\right) N\right]^{1 / 2}\right\} \\
& \times \exp (i m \phi) \exp \left(-i \frac{n}{z_{0}} z\right),
\end{aligned}
$$

where $c(n, m)$ is constant for each mode and $\xi_{1}{ }^{2} \equiv$ $n_{1} /\left(\pi^{2} w_{0}^{2}\right)$. The two-dimensional Fourier transform of Eq. (22) over the transversal coordinates $(\rho, \phi)$ satisfies

$$
U_{n, m}^{\operatorname{limit}}(\xi, \Theta) \propto \exp \left[-\pi\left(w_{0} \xi\right)^{2}\right] * \operatorname{ring}_{N, m}(\xi, \Theta),
$$

where $*$ denotes convolution, $(\xi, \Theta)$ are the polar spatial-frequency coordinates and

$$
\operatorname{ring}_{N, m}(\xi, \Theta) \equiv \delta\left\{\xi-\left[\xi_{1}^{2}+(2 / \lambda \Delta z) N\right]^{1 / 2}\right\} \exp (\operatorname{im} \Theta) \text {. }
$$

As the Gaussian spot size increases, that is, $w_{0} \rightarrow \infty$, the convolution kernel in Eq. (23a) becomes infinitesimally narrow. In this limiting case the SI solutions are in agreement with Ref. 5, and their spectrum is concentrated about the rings described in Eq. (16).

Note that $\xi_{1}{ }^{2}$ can take any positive real value leading to any positive ring radius. However, to contain fully the solutions of Eq. (16), it should also be possible to obtain $\xi_{1}=0$, corresponding to a plane wave. In this special case, the basic Gaussian mode $\left(n_{1}=0\right)$ can be introduced and considered as a plane wave. For this purpose we use a different limit: Condition (B) applies only for $j>1$, and condition (C) is changed to $\tilde{\rho} \ll 1 / 2$.

It should be pointed out that some of the conditions above may not seem necessary for simulating the infinite effective-aperture situation. For example, Eq. (19) was chosen to fit all modes into Eq. (21). However, here we are interested only in showing that under sufficient conditions the solutions of Eq. (16) are a limiting case of Eqs. (10) and (11).

In contrast with previous research, ${ }^{4-9}$ our approach deals with waves that are exact solutions of the paraxial wave equation. No further approximations are made. The GL modes fall off rapidly away from the propagation axis and thus limit the support of the object to an effective finite aperture. Truncation effects can be arbitrarily reduced, producing negligible aberrations in the results. Note that the GL modes can be expressed as superpositions of GH modes having $n_{x}+n_{y}=n$ and vice versa. ${ }^{12}$ Thus Eqs. (9) and (11) also apply for wave representations in terms of $\mathrm{GH}$ modes, with $n$ defined in this way.

In summary, scaled SI can exist between any two planes and with any magnification within the paraxial regime. In an appropriate GL basis, Eqs. (10) and (11) give a necessary and sufficient condition for SI. The phase map, scaled with the corresponding Gaussian spot size, is also replicated with the exception of a quadratic phase. These waves possess finite energy and thus are physically realizable. Previously known SI wave fields are obtained as a particular limiting case. The theoretical results presented here may prove useful for the analysis and synthesis of waves.

This study was started while Y. Y. Schechner was at the Faculty of Physics, Technion-Israel Institute of Technology. The research was partially supported by the Philipson Fund for Electrical Power and by the Fund for the Promotion of Research at the Technion (Research No. 050-853).

\section{References}

1. G. Indebetouw, J. Opt. Soc. Am. A 6, 150 (1989).

2. G. Indebetouw, J. Mod. Opt. 40, 73 (1993).

3. Y. Y. Schechner, R. Piestun, and J. Shamir, Phys. Rev. E 54, R50 (1996).

4. W. D. Montgomery, J. Opt. Soc. Am. 57, 772 (1967).

5. W. D. Montgomery, J. Opt. Soc. Am. 58, 1112 (1968).

6. J. M. Cowley and A. F. Moodie, Proc. Phys. Soc. B 70, 505 (1957).

7. A. Kalestynski and B. Smolinska, Opt. Acta 25, 125 (1978).

8. A. P. Smirnov, Opt. Spectrosc. 44, 208 (1978).

9. K. Patorski, in Progress in Optics, E. Wolf, ed. (NorthHolland, Amsterdam, The Netherlands, 1989), Vol. XXVII, pp. 3-108.

10. P. Szwaykowski and J. Ojeda-Castaneda, Opt. Commun. 83, 1 (1991).

11. M. Abramowitz and I. A. Stegun, eds., Handbook of Mathematical Functions (Dover, New York, 1965).

12. G. Nienhuis and L. Allen, Phys. Rev. A 48, 656 (1993). 